\title{
As Características do Desenvolvimento Distribuído de Software
}

Fábio Levy Siqueira, Paulo Sérgio Muniz Silva

Departamento de Engenharia de Computação e Sistemas Digitais - Escola Politécnica da Universidade de São Paulo

Av. Prof. Luciano Gualberto, trav.3, n 158 - 05508-900 - São Paulo - SP - Brazil

\{levy.siqueira, paulo.muniz\}@poli.usp.br

Abstract. The distributed software development has different forms, each one generating benefits, problems, and solutions. This paper proposes an abstraction of the distributed software development into a set of characteristics, in order to highlight the differences of this kind of software development when considering the main problems pointed out by the current research. Three case studies discussing how these characteristics are differently instantiated in software development projects are presented.

Resumo. Existem diversas formas do desenvolvimento distribuido de software, cada uma delas levando a diferentes beneficios, problemas e soluções. Nesse trabalho é proposta uma abstração do desenvolvimento distribuido de software em um conjunto de características, buscando evidenciar as diferencas dessa forma de desenvolvimento ao considerar os principais problemas apontados pela pesquisa atual. A partir disso são apresentados tres estudos de caso que alisutem como escas caracteristicas san diferentemente instanciadas em projetos de desenvolvimentos de software.

\section{Introdução}

Um desenvolvimento de software é tradicionalmente realizado com pessoas localizadas lado-a-lado, o que permite um fluxo constante de informações e idéias (Cockburn, 2002). No entanto, em algumas situações esse formato de solução pode não ser o mais viável. A complexidade de um projeto pode obrigar que ele seja realizado por diversas empresas espalhadas por uma mesma cidade; a busca por um especialista, seja ele uma pessoa ou uma organização, pode levar a outros estados; a necessidade competitiva de cortar custos ao utilizar mão-de-obra barata e ainda assim qualificada pode envolver organizações em outros países. Nesses casos em que as pessoas envolvidas no projeto não podem trabalhar em proximidade física é necessário realizar uma outra forma de desenvolvimento: o desenvolvimento distribuído de software (DDS)

Assim como existem diversas vantagens para a realização, existem diversas dificuldades e problemas ao realizar o desenvolvimento de software dessa forma. A experiência prática mostra que a separação espacial e temporal das pessoas afeta profundamente o desenvolvimento de software, tornando mais difícil a sua realização devido a inúmeros motivos que variam desde a ausência de um idioma e uma faixa de horário comum a até a falta de confiança e de senso de equipe entre as pessoas envolvidas (Carmel, 1999); (Mockus e Herbsleb, 2001). 
Por mais que existam inúmeras vantagens e problemas ao realizar desenvolvimento distribuído de software, é importante notar que muitos dos atrativar un dificuldades estão diretamente ligados a alguns formatos do DDS. A abrangência des e termo engloba desde casos em que as pessoas estão distribuídas em grupos localizado em diferentes prédios de uma mesma cidade, a até situações em que os desenvolvedos estão completamente dispersos pelo mundo. Só ao observar essas duas possibilidare possível notar que o DDS tem diversas facetas, levando a diferentes bendes, problemas e soluções que farão, por exemplo, com que os processos empregados, riscos e a estratégia da gerência sejam completamente diferentes. Dessa forma, antes de criar soluções é preciso entender o que o DDS significa para um determinado projeto observando as principais fontes de possíveis problemas.

Para denotar as diferenças dessa forma de desenvolvimento, nesse artigo é apresentada uma proposta de abstração do DDS em um conjunto de característica Espera-se com isso facilitar a observação das origens dos problemas e das vantagens que existem em desenvolvimentos dessa forma, buscando também um melhor entendimento de como as atividades relativas à engenharia de software são afetadas. Para isso, o artigo está estruturado da seguinte maneira: na seção 2 é apresentada a base teórica ao discutir alguns trabalhos relacionados. Na seção 3 é apresentada a proposta de abstração do DDS em características, descrevendo alguns problemas. Para discutir como essas características existem de forma diferente em projetos DDS, na seção 4 são apresentados três estudos de caso. Por fim, na seção 5 é apresentada a conclusão, abordando algumas considerações finais e perspectivas de trabalhos futuros.

\section{Trabalhos Relacionados}

Alguns autores já abstraíram em diferentes formas e com diferentes profundidades 0 DDS a um conjunto de características (ou dimensões). Ao criar uma proposta de classificação, alguns trabalhos apontam indiretamente as características consideradas mais importantes. Cockburn (2002), por exemplo, considera basicamente o tamanho da equipe e os papéis existentes em cada local, além do tipo do projeto (código aberto ou comercial) para realizar a sua classificação em 4 tipos de DDS (multisite, offshore, distribuído e software open-source). Para definir o que é DDS, Prikladnicki et al. (2003) relevam para uma classificação a separação física interna e externa a alguns grupos de partes envolvidas no projeto.

Uma vista mais direta à questão é feita por Carmel (1999) que aponta o que diferencia o desenvolvimento global de um tradicional: a distância física, o fuso horário e a cultura nacional. No entanto, a caracterização em três dimensões parece ser muito restrita, o que pode ser observado em alguns trabalhos que consideram uma maior variedade de características. Kobitzsch et al. (2001), por exemplo, afirmam existirem diversos fatores que diferenciam o DDS, mas para a criação de seu modelo são apenas considerados os que seriam as mais importantes: a relação legal e a configuração da equipe.

Um tratamento multidimensional mais exaustivo e que tenta obter uma divisão mais geral ao assunto é proposto por Evaristo e Scudder (2000). Essa divisão tem como foco a gerência de projetos e busca as variáveis que influenciam no desempenho do projeto, levando em consideração as seguintes dimensões: o tipo de projeto, a estrutura do projeto, a distância percebida, a sincronia entre os trabalhadores, a complexidade do diferenças culturais, a existencia de diversas metodologias, a diversidade e projeto, as de consenso de políticas e padrões, o nível de dispersão entre grupos de pificulado de diferenca de interesses das partes envolvidas. Como pres envolvidas e a quantidade é p a preoctando algumas características que não são relativas apenas à forma como as consideran estão distribuídas, observando como ocorre o desenvolvimento e também a pessoas esta pelo projeto envolver um grande contingente de pessoas.

\section{Desenvolvimento Distribuído de Software}

3. Abstração do Desenveristintos, o que remet As diversas vistas para as caracterist Como o objetivo das características aqui propostas às diferenças de idéias apresentadas. Cono o ObS for evidenciar as diferenças entre projetos DDS fo éviden diversas idéias. Mais que isso, foram também considerados foram cons relatos de experiências e discussões sobre o assunto. A partir disso foram diversos relatos dex desse tipo e as observados os principais problemas existentes em desenvolvimentos desse tipo e as diversas opções possíveis de DDS, chegando a essa abstração.

As características do DDS aqui propostas são provenientes de três categorias principais: a forma de separação dos grupos (agrupamento, distância física e separação principais: a regiões envolvidas (culturas regionais, idiomas e diferenças dos locais) temporal), as regióes envolvidas (culturas regionais, idiomas e diferenç também as organizações participantes (culturas organizacionais, infrastrura relação legal). O ponto crítico do desenvolvimento distribuído de software, que também relaçáo lé separação dos grupos. No entanto, essa separação física acaba o define, é a forma de separação dos grupos. No entanto, essa separaçá estejam em levando aos outros dois pontos, já que é bastante comum que as pessoas estejam en outras regiões (cidades, estados e países diferentes) e também que os grupos separados geograficamente façam parte de organizações diferentes. E como esses doi
pontos são imprescindiveis para entender o DDS, eles foram aqui considerados.

A seguir é apresentada cada uma das características, observando o que cada uma delas representa e alguns dos problemas mais comuns gerados em um DDS.

\subsection{Agrupamento}

A idéia principal contida no DDS é que as pessoas envolvidas não estão lado-a-lado, mas isso não significa que obrigatoriamente todas as pessoas estão separadas umas das outras. Uma opção bastante comum de DDS é o caso em que as pessoas estão distribuídas em grupos, trabalhando lado-a-lado com algumas pessoas e de forma distribuída com outras.

A forma como o grupo está distribuído parece ser uma questão multidimensional composta de três aspectos: a quantidade de pessoas no grupo, a quantidade de grupos e os papéis exercidos pelas pessoas. Dependendo da mescla desses aspectos torna-se possível montar grupos auto-suficientes (analistas, programadores, testadores, etc), diminuindo a necessidade de comunicação feita entre os grupos (Kroll e Kruchten, 2003)

3.2. Distância física

Conforme a separação física das pessoas aumenta, torna-se cada vez mais dificil realizar reuniões presenciais entre os membros do projeto, algo que pode ser importante durante 
alguns momentos do projeto. McGrath (1990 apud Herbsleb e Mockus, 2003) s que os grupos forçados a se comunicarem primariamente através de telecomunigere terão maior dificuldade em alguns estágios de uma tarefa, como a solução de problợes técnicos e a resolução de conflitos.

Realizar uma viagem para encontrar as outras pessoas pode ser demasiadamente custoso, demorado ou cansativo, o que pode dificultar significativamente a realizinte de reuniões presenciais. Mas além de dificultar a realização destas reuniões, a disaçào física também pode ocasionar alguns outros problemas. A comunicação entre as pesta pode sofrer com a defasagem na transmissão de informações, causando inconveniente durante uma vídeo-conferência ou uma conversa por telefone como, por exemplo existência de duas pessoas falando ao mesmo tempo e a dificuldade de saber quando pessoa terminou de falar.

\subsection{Separação temporal}

Dependendo da distância entre os locais, a separação espacial acaba trazendo também uma separação temporal através das diferenças de fusos horários (principalmente em desenvolvimentos globais), que diminuem as intersecções nos horários de trabalho entre pessoas de grupos diferentes. Mas além da questão do fuso horário, a separação temporal pode também existir com uma diferença de horários de trabalhos entre as pessoas de um mesmo local, como, por exemplo, na existência de diferentes turnos de trabalho - um caso limite do DDS em que as pessoas trabalham em um mesmo local, mas em horários diferentes.

Independente de como a diferença de horários é ocasionada, essa característica leva a diversos problemas no desenvolvimento de software ao dificultar uma troca de informações síncrona - o que pode ser uma necessidade dependendo da urgência e do tipo de discussão realizada. Uma dúvida que apareça pode levar diversas horas para que seja sanada ao ser necessário esperar por uma determinada pessoa. Por outro lado, separação temporal pode ser positivamente aproveitada ao organizar o trabalho para um desenvolvimento durante 24 horas (Haywood, 2000).

\subsection{Culturas regionais}

Um dos problemas comumente apontados, principalmente em casos de desenvolvimento global, é o aspecto cultural. Esses problemas ocorrem por diferenças de comportamentos entre pessoas de diferentes culturas como, por exemplo, diferenças no planejamento do trabalho, no processo decisório, no estilo de argumentação, no fluxo da conversa, entre outras (Olson e Olson, 2003). Mas é importante ressaltar que essas diferenças não são privilégios de desenvolvimentos globais, já que até pessoas de um mesmo país podem sofrer com problemas culturais devido às diferenças regionais.

\subsection{Idioma}

Por mais que o idioma seja uma das manifestações da cultura, a sua extrem importância durante um desenvolvimento de software (ou qualquer atividade realizad em grupo) faz com que ele se sobressaia entre as demais diferenças culturais. Mesmo que os grupos tenham costumes e crenças completamente diferentes, com um idiom comum entre as partes é possivel desenvolver um sistema - por mais que ocorram problemas e conflitos -

o contrário parece pouco factível, ja que as pessoas mal

No desenvolvimento de software, alem da comuch com que a grande ainda existem os diversos documentos de projeto. Isso faz cor todas as partes em geral, adieto maioria dos projetos adsim, alguns problemas continuam a ocorrer pela falta de nvolvidas. Mesmo assim,

proficiência de alguns membros eãa e cultura (Mockus e Herbsleb, 2001).

\section{Diferenças dos locais} A localização de um dos groblemas que melhor diversas formas o desenvolvimentos locais sejam os aspectos jurídicos. Dois grupos videnciam a diferença entre os locais sejam os aspectos jum elas comerciais, evidiferentes países estarão sujeitos a diferentes leis, sejam elas comerciais, localizados ens diferentes legislações podem afetar de diversas formas o trabalhistas, civis, etc. Esas dire, como, por exemplo, ao dificultar (ou até impedir) a dware (Haywood, 2000), ao necessitar diferenças importação de um determinado hardware (Hayso para viagens de

contratuais como o ano fiscal ou

Além da questão jurídica, o local pode ter alguns outros problemas ou simples ferencas que acabam de alguma forma dificultando o desenvolvimento de software e for Kobitzsch et al. aumentando o custo de investimento. Em um caso apresentado levando à compra de (2001), a volatilidade da infra-estrutura de um local acabou levano eradores e também a compra de linhas independentes de comunicação.

\subsection{Culturas organizacionais}

No desenvolvimento de software as diferenças de culturas organizacionais podem os diversos entendimentos do que significa realizar causar alguns problemas com che gar a um consenso de testes de unidade (Mockus e Herbsleb, 2001), a difisão diferente do que é qual seria o uso sistema (Damian e Zowghi, 2003) e a visa qualidade (Kobitzsch et al., 2001).

É curioso observar que alguns dos problemas ot à culturas regionais podem tambem aparecer devido as culturas organizacionais. Schein (1992) cita, por exemplo, diferenças no comportamento frente à hiso demonstra orientação ao processo entre organizações de um mesmo pais. Isso dé fazer com complexidade da relação entre as diversas formas da cultura, o que pode até fazer coma que alguns dos problemas que são freqüentemente apontados por uma deter

cultura regional não existam devido a uma determinada cultura organizacional.

\subsection{Infra-estrutura das organizações}

Qualquer organizacão que desenvolve software, independente se realizando um DDS ou não, precisa de uma envolvidas. Como infra-estrutura entende-se o hardware, o software, as ferramentas, as técnicos operação e manutenção de produtos de software (ABNT, 1998). 
Em um DDS a existência de infra-estrutura adequada por todas as envolvidas pode ser difícil de se obtida. Mesmo dentro de uma organizacã as par problemas pelo fato de seus funcionários trabalharem em casa, em hotéiso pode ha locais. Quando são envolvidas mais de uma muito maior. As organizações podem trabalo dificuldade se totros padrões de código, ambientes de torna tornar a coexistên de pros umes enorme desafio. problema pode não ser exatamente o conflito na infa-estrutra, Mas muitas vezes infra-estrutura adequada - um aspecto funder alta de 0 infra-estrutura de telecomunicações adequada (Carmellara o DDS é a existência de uma

\subsection{Relação de negócio}

Em projetos de DDS é bastante comum a existência de diversas organzaços trabalhando em conjunto, dividindo de aloum form organizaçõe Essas organizações podem ser ligadas através desenvolvimento terceirização), ou fazerem parte de uma mesma uma contratação (como em uma filial), ou até alguma outra forma (como uma matriz e um forma uma nova entidade (Karolak, 1998)), por exemplo. (relação entre empresas que

Dependendo da natureza do

diferenças e problemas específicos no relações, em um projeto existirão algumas (1999), a relação de negócios é coesa. Além disso a formação de uma equipe conhecimento entre as a pagem de pode não proprietária, mas que podema compartilhar algumas informações que ela considera

\section{Estudos de caso}

Cada DDS apresenta diversas diferenças, que podem fazer com que o problema de um determinado projeto seja irrelevante para um outro. A abstração em um conjunto de características aqui proposta busca organizar esses problemas a partir de suas origens para facilitar a observação do que é mais importante para o projeto.

Para discutir como essas características existem na realidade, serão apresentados projeto A foi um projeto acadêmios (ou sendo realizados) de forma distribuída. 0 feito por pessoas que se encontrava fra a construção de um software de colaboração $\mathrm{O}$ projeto $\mathrm{B}$ está $\mathrm{em}$ an de um software de um software da área de astrofísica. O projeto $\mathrm{C}$ foi um grande projeto de uma grande into de um framework para a construção de aplicações financeiras para DDS estão instição financeira. Na Tabela 1 é apresentado como as características do

(ou afetam) o projeto.

Através da tabela é possível observar que, apesar dos três projetos serem desenvolvimentos distribuídos de software, que, apesar dos três projetos serem completamente diferentes. No projeto A dificuldades foram (e são) pequenas mudancas no no empregado e na infra-estrutura utilizada, enquanto que enquanto que diversas dificuldades, como conflitos de infra-estrutura, diferentes filosofias e objetivos diferentes culturas organizacionais) e a quase inviabilidade de realizar reuniões pelas ciais devido à dispersão das pessoas e a falta de recursos, por exemplo. Mais presencíático ainda, no projeto $\mathrm{C}$ praticamente cada característica gerou algum tipo de Alguns desses problemas são semelhantes ao do projeto $\mathrm{B}$, como as problerca Alguns difticularidades que geram outros problemas, como, por exemplo, a cultura regional e o particula que causaram conflitos e mal entendidos, e o agrupamento que, apesar de dificultar as reuniões presenciais assim como no projeto B, influiu primariamente na discussão entre diferentes grupos de partes envolvidas.

Tabela 1. As características observadas nos projetos e o quanto elas o afetam.

\begin{tabular}{|c|c|c|c|}
\hline & Projeto A & Projeto B & Projeto C \\
\hline Agrupamento & $\begin{array}{c}4 \text { pessoas dispersas ( } 3 \\
\text { desenvolvedores e } 1 \\
\text { gerente) }\end{array}$ & $\begin{array}{c}5 \text { locais com } 1 \text { a } 3 \text { pessoas } \\
(9 \text { pessoas no total), sem } \\
\text { qualquer forma de divisão } \\
\text { dos papéis }\end{array}$ & $\begin{array}{c}3 \text { locais com } 25 \text { a } 30 \\
\text { pessoas ( } 80 \text { pessoas no } \\
\text { total), com divisão por } \\
\text { papéis }\end{array}$ \\
\hline Distância física & $\begin{array}{l}\text { Mínima: dentro de uma } \\
\text { mesma cidade e se viam } \\
\text { freqüentemente }\end{array}$ & $\begin{array}{l}\text { Razoável: em estados do } \\
\text { Brasil, mas sem recursos } \\
\text { para viagens freqüentes }\end{array}$ & $\begin{array}{l}\text { Grande: um local no } \\
\text { exterior e dois em estados } \\
\text { brasileiros, com recursos } \\
\text { para viagens freqüentes }\end{array}$ \\
\hline $\begin{array}{c}\text { Separação } \\
\text { temporal }\end{array}$ & Inexistente & $\begin{array}{l}\text { Possível diferença nos } \\
\text { horários de trabalho }\end{array}$ & $\begin{array}{l}\text { Grandes diferenças de } \\
\text { fuso-horário }\end{array}$ \\
\hline $\begin{array}{l}\text { Culturas } \\
\text { regionais }\end{array}$ & Sem diferenças & $\begin{array}{l}\text { Pouca diferença } \\
\text { (regiões do Brasil) }\end{array}$ & $\begin{array}{l}\text { Muita diferença } \\
\text { (diferentes países) }\end{array}$ \\
\hline Idioma & Igual & $\begin{array}{c}\text { Diferença mínima } \\
\text { (diferença regional) }\end{array}$ & $\begin{array}{c}\text { Grandes diferenças } \\
\text { ( } 2 \text { idiomas })\end{array}$ \\
\hline $\begin{array}{c}\text { Diferença dos } \\
\text { Iocais }\end{array}$ & Praticamente nenhuma & Praticamente nenhuma & Grandes diferenças \\
\hline $\begin{array}{c}\text { Cultura } \\
\text { organizacional }\end{array}$ & Pequena diferença & $\begin{array}{c}\text { Diferente: várias } \\
\text { organizações envolvidas } \\
\end{array}$ & $\begin{array}{c}\text { Diferentes: duas } \\
\text { organizações envolvidas }\end{array}$ \\
\hline Infra-estrutura & Pouco diferente & $\begin{array}{c}\text { Diferente e com } \\
\text { dificuldade para acertar }\end{array}$ & Pouco diferente \\
\hline Relação legal & $\begin{array}{l}\text { Pouco influiu: projeto } \\
\text { acadêmico }\end{array}$ & $\begin{array}{c}\text { Convênio entre } \\
\text { universidades e trabalho } \\
\text { voluntário }\end{array}$ & $\begin{array}{l}\text { Parceria entre as } \\
\text { organizações }\end{array}$ \\
\hline
\end{tabular}

\section{Conclusão}

O desenvolvimento distribuído de software é um termo bastante abrangente. Diversas situações são possíveis, cada uma com diferentes problemas a serem gerenciados, benefícios a serem aproveitados e soluções a serem estudadas e aplicadas. Nesse artigo foi apresentada uma abstração do DDS que busca facilitar o entendimento do que significa essa forma de desenvolvimento e quais são as origens dos problemas e das vantagens existentes no DDS.

Para a apresentação dessa abstração, pretendeu-se neste artigo descrever brevemente cada uma das características propostas, apontando alguns problemas causados. Em trabalhos futuros pretende-se detalhar os problemas e vantagens que são originários de cada característica, refinar a observação da influência das características (criando uma gradação objetiva), além de propor algumas soluções para os problemas mais comuns (com uma preocupação na área de gerência de projetos). Com isso, se espera obter uma maior maturidade nessa abstração, o que talvez obrigue considerar 
algumas outras possibilidades de características ou até a desconsideração de outros, almejando criar um conjunto suficiente e que demonstre a essência do DDS.

\section{Agradecimentos}

Essa pesquisa foi financiada por uma bolsa de mestrado fornecida pela Escola Politécnica da Universidade de São Paulo e pela CNPq.

\section{Referências}

ABNT - Associação Brasileira de Normas Técnicas. NBR ISO/IEC 12207 - tecnologia de informação: processos de ciclo de vida de software. ABNT, 1998.

CARMEL, E. Global Software Teams: Collahorating Across Borders and Time Zones. Prentice Hall, 1999

COCKBURN, A. Agile Software Development. Addison Wesley, 2002.

DAMIAN, D.; ZOWGHI, D. An insight into the interplay between culture, conflict and distance in globally distributed requirements negotiations. In: Hawaii International Conference On System Sciences, 36., Hawaii, 2003.

EVARISTO, J. R.; SCUDDER, R. Geographically Distributed Project Teams: A Dimensional Analysis. In: Hawaii International Conference On System Sciences, 33., Hawaii, 2000.

HAYWOOD, M. Working in Virtual Teams: A Tale of Two Projects and Many Cities. IT Professional, v.2, n.2, p.58-60, March/April 2000.

HERBSLEB, J. D.; MOCKUS, A. An Empirical Study of Speed and Communication in Globally Distributed Software Dévelopment. IEEE Transactions on Software Engineering v.29, n.6, p.481-494, June 2003.

KAROLAK, D. W. Global Sofiware Development: Managing Virtual Teams and Environment. IEEE Computer Society Press, 1998.

KOBITZSCH, W.; ROMBACH, D.; FELDMANN, R. L. Outsourcing in India. IEEE Software, v.18, n.2, p.78-86, March/April 2001.

KROLL, P.; KRUCHTEN, P. The Rational Unified Process Made Easy: a Practitioner's Guide to the RUP. Addison-Wesley, 2003.

MOCKUS, A.; HERBSLEB, J. D. Challenges of Global Software Development. In: International Software Metrics Symposium, 7., London, 2001.

OLSON, J. S.; OLSON, G. M. Culture Surprises in Remote Software Development Teams. Queue Focus: Distributed Development, v.1, n.9, p.52-59, December/January 2003-2004, 2003.

PRIKLADNICKI, R.; AUDY, J.; EVARISTO, R. Distributed Software Development: Toward an Understanding of the Relationship Between Project Team, Users and Customers. In: International Conference On Enterprise Information Systems, 5., Angers, 2003.

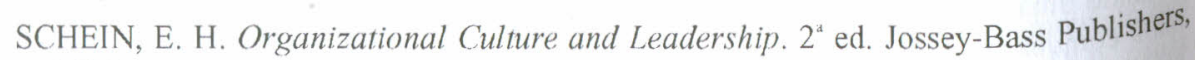
1992. 